\title{
Radiating Centers: Augmented Reality and Human-Centric Design
}

\author{
Isabel Pedersen ${ }^{1}$ \\ Department of Professional Communication \\ Ryerson University, Canada
}

Published: Isabel Pedersen. "Radiating Centers: Augmented Reality and Human-Centric Designs" IEEE International Symposium on Mixed and Augmented Reality (ISMAR). Orlando, Florida, October 19-23, 2009.

\begin{abstract}
People understand themselves as centers. Using a humanities approach, this paper proposes a conceptual model that promotes human-centricity for AR experiences. It uses David Burrows' phenomenology of sound and human thought as a reflective model. He argues that humans act according to a center-periphery scheme that is projected in three fields of action relating to body, mind, and the existential self. The paper suggests that AR inventors ought to consider how humans imagine their own centric position in the world when they design new applications and interfaces. The thesis for this short paper is that a humanitiesbased vision for AR could assist with overcoming the disjuncture between the real and the virtual at the early design stages and make human participants feel more integrated.
\end{abstract}

KEYwORDS: interactivity and interaction, human-centric design, humanities approach

INDEX TERMs: H.5.1 [Information Interfaces and Presentation]: Artificial, augmented, and virtual realities; H.5.2 [User Interfaces]: User-centered design

\section{INTRODUCTION}

William Gibson's novel, Spook Country (2007) [1] offers a utopian vision of Augmented Reality (AR). In the book, locative artists upload art installations to servers and leave them at locations for underground art enthusiasts to enjoy. Cell phones and GPS units hacked together with duct tape form one device. Others require goggles. At one point, a character wades through a room filled with Monet's poppies so seemingly real she is sure that she can actually feel them. However, the book frames this idyllic depiction of augmented reality art in a dystopia that involves organized crime, drug addiction, heightened state surveillance, and the aura of post 9/11 society. Personal mobile devices like iPods so normally associated with individual liberation and enjoyment are used for smuggling information and leveraging criminal activity. The New York Times book reviewer for Spook Country focuses on ironic disjuncture in the novel and entitles his review "Spirits in a Material World" [2]. He is referencing the fact that each of the three main characters is a "spirit," a "novice initiated into an alternative reality he or she never knew existed" [2]. One is a former member of a cult rock band, now turned journalist, writing for a magazine that might not exist. Another is a young immigrant drawn into a secret familybased society that has given him a mission which could alienate him forever from his previous life. And the last is an Ativan drug

1 ipederse@ryerson.ca addict captured and forced to help a man, who may or may not be a government agent, with a dangerous case. The common thread across all of these characters is that their current predicament utterly displaces them from their regular lives. All of them are completely confused about their roles, even though they have been given tasks to fulfill. Much of the time, they act blindly. Augmented reality becomes a metaphor for these characters who cannot properly contextualize themselves in their surrounding new worlds; their disconnected existence seems virtually fabricated. They face conflicting identities.

However, these characters, as a core, also serve as a metaphor for augmented reality itself, a medium of communication that labors with the ultimate goal of merging a real and a virtual reality. While AR technology has progressed to impressive levels, it needs to be envisioned as a medium embedded in the lives of humans. It needs to work within contexts that are meaningful to humans, who are embodied and signifying agents in the world.

Using a humanities approach, this paper offers a conceptual design strategy, a set of ideas. It suggests that human users need to be treated conceptually as centers (i.e., human-centric positioning), immersed in AR interfaces, rather than as viewers seeing interfaces or graphical overlays, even if the actual technology is about seeing a virtual component. While there are many ways to conceive of and model human-centricity, this paper offers a unique approach using David Burrows' phenomenology of sound and human thought [3] as a reflective model. It suggests three complementary ideas to be embraced at the early design stage in pursuit of human-centricity:

(1) Center the human participant -- AR interfaces ought to be designed according to a center-periphery schema, which will better enable humans to feel connected and contextualized in real and virtual realities.

(2) Leverage sound as a concept -- AR designers should consider the idea of sound as much as they do the idea of sight when conceptualizing human-computer interaction for applications.

(3) Acknowledge that human participants move, think, and exist simultaneously -- AR interfaces need to accommodate human participants who not only act physically, but also act mentally, and existentially (constantly negotiating the self in relation to others and the physical world).

A humanities-based approach offers a researcher the opportunity to address concepts and ideas. It enables one to ask questions like How are we persuaded to adopt an interface? How do films or books alter our real-world approaches to designing new media? Or How do aspects of our humanity, the ways that we think, feel, sense, and communicate, bear upon the ways that we approach technology?

Many people have written about mixed and augmented reality in a humanities context [4] [5] [6] [7] [8] [9] [10] [11]. Some technologists have offered a human-centric design approach for computer interfaces. Indeed, Ben Shneiderman writes in Leonardo's Laptop that "if technology developers start from an understanding of human needs, they are more likely to accelerate evolutionary development of useful technology" [12]. He offers a 
conceptual approach to need that ranges far beyond notions of it as basic human sustenance. In similar fashion, this paper first takes an original approach by using the theory of David Burrows described in his book Sound, Speech, and Music [3] to serve as a conceptual model for human-centricity in interfaces. Second, it will reflect on some new AR applications in light of the theoretical model to foreground how human centricity can be achieved. Third, it will speculate about some future AR applications in light of the first two goals. The thesis for this paper is that a humanities-based vision for AR could assist with overcoming the disjuncture between the real and the virtual at the early design stages and make human participants feel more integrated.

\section{THEORY}

Because of its nature, AR is far more bound up in the everyday dealings of human life than most other computing media. Augmented reality implies that users are interacting with real life and a virtual reality at the same time. Unlike the medium of virtual reality, augmented reality users physically range outside of "protected" environments like virtual reality CAVEs®. They are subject to all the delights and dangers of the real world, in addition to an augmented, virtual world. Azuma [13] and Azuma et. al. [14] offer a broad definition of augmented reality in three criteria: it "combines the real and virtual;" it is "interactive in real time;" and it is "registered in three dimensions" [13] [14]. Even though AR is defined in flexible, conceptual terms, the one concrete goal for it as a medium of communication is that the virtual aspect must cohere with the real world of humans. There are technologists all over the world concerned with technical coherence, or making sure the virtual and real register correctly. This paper deals with how humans imagine themselves cohering with the world, with reality, in the first place. It suggests that by basing AR upon a conceptual foundation that explains how humans act and think about acting, we can achieve more integrative AR applications.

David Burrows refers to his work, at one point, as a "phenomenology of sound, speech, thought, and music" [3]. Phenomenology is a broad field of the humanities that explores aspects of our personal sense of existence, our consciousness. It gives us a way to consider how each person has a unique point of view. Traditionally, writers associated with it include Edmund Husserl, Maurice Merleau-Ponty, and Martin Heidegger. Today, many writers use phenomenology to analyze human computer interaction and to influence design including Michael Heim [15], Richard Coyne [16] and Paul Dourish [17]. Dourish, for example, uses Merleau-Ponty's notion of embodiment, to develop a theory of "embodied interaction” for our era of ubiquitous computing [17].

However, Burrows does not address computers, AR, or any sort of technology (beyond musical instruments) in his writing. Rather, like many philosophers, he offers an argument concerning the unique aspect of human beings. He claims that the distinctiveness of "human beings" - that which sets them apart from other species - is their capacity for "free-wheeling and wide-ranging thought" [3]. For Burrows, thought makes human beings distinctive. Further, he writes that human thought is "an outgrowth of the distinctive capacity of the way [humans] use sound, itself distinctive in a number of ways among the senses" [3]. He argues that human thought is a by-product of our experience with sound. In other words, hearing teaches us thinking. Further, he claims we neglect sound in a world dominated so much by sight. He writes that "for most people most of the time, sound is one of the unconsidered ubiquities, as transparent to scrutiny as the air that carries it” [3].
Burrows makes one additional claim to underscore his phenomenology. He states that the way we hear (and think) is governed by a center-periphery scheme. Burrows is clearly not the first to embrace the scheme as an essential aspect of humanity. The famous gestalt psychologist, Rudolph Arnheim argues that "the centric stands for the self-centered attitude that characterizes the human outlook and motivation at the beginning of life and remains powerful throughout" [18]. Burrows points directly to cognitive linguist Mark Johnson who writes about the centerperiphery scheme in his work The Body in the Mind [19]. Johnson identifies center-periphery as one of the schema central to our framing of language. He develops his notion of bodily schema from his first book with George Lakoff, Metaphors We Live By [20]. However, Burrows takes an original stance on the scheme because he combines center-periphery with sound. He describes the way it operates:

[the scheme] characterizes the individual, living human body as a center with a strong instinctual tendency to treat everything else in its cosmos as peripheral to it . . . Life is seen here as a radiation around a center. The further it radiates - the more territory it controls - the more secure it feels (but the center/periphery scheme applies not only to relations between the body and its surroundings; it organizes the interior world of the body itself as well). This means that the centripetal/centrifugal coordinate of action, governing motion toward and away from the center is fundamental, and the self is defined whenever the force of its centrifugally radiating energies encounters the resistance of the "other." [3]

This passage's key phrase is "Life is seen here as a radiation around a center." Life radiates. Human action involves pulling (centripetal) and pushing (centrifugal) away from a conceptual center. This trajectory of action defines the self, not only physically, but also mentally and existentially. For Burrows, the center-periphery scheme is fundamental to all human action and we can acknowledge it by understanding the way sound (and our ability to hear) centers us.

Burrows goes on to characterize the ways humans act according to a notion of centricity. The center-periphery scheme is projected in three fields of action relating to body, mind, and the existential or spiritual self [3]. Field 1 governs the body, which is mobile within a physical space. In this field, the center is the physical body and the periphery involves separation and connection with other things and people in the world. Field 2 is the "invisible and intangible space where thought takes place” [3]. It signifies a mental space where ideas range across past, present, future, and elsewhere no longer rooted to the physical of Field 1 . The constraints of Field 1 involve the here and now; they are physical limits. However, concepts like logic, clarity, and the intelligibility of the speech act govern Field 2. Finally, Field 3 is the field relating to existence, "the sense of self diffused through the full range of awareness. . . .whose center . . . 'is everywhere and the periphery nowhere", [3]. In Field 3, humans operate without constraints and "action tends to be suspended or to assume cyclic, self-enfolded forms” [3]. In Field 3, participation in music, ritual, and meditation abounds. Overall, Burrows argues that Fields 2 and 3 liberate human thought through the capacity of sound (specifically vocal sound), which detaches humans from the physical world, or the constraints of Field 1 where sight is so dominant. Humans act simultaneously across the three fields. In mundane terms, this process presents human action as a totality. Burrows gives us a set of terms to explain that the way we move in the world (e.g., on land, in buildings), coincides with our social 
interaction and mental interaction, and that the two are intimately intertwined with the way we act as selves.

The next few paragraphs describe these three fields more specifically. Further, it goes on to use Burrows' philosophy as a conceptual model for designing AR user experiences. This paper also offers some exemplars from existing AR technology to bring design ideas to the fore.

\subsection{Physical Activity}

Burrows describes physical action within Field 1:

Field 1 is physical space, the space taken up by the body and through which the body moves, the space surveyed by the senses, with vision bearing the heaviest responsibility among them. In this Field, the center is always identified with the physical body. It takes precedence over the other fields to the extent that the physical survival of self is at issue. ...

Movement makes degrees of solidity a central consideration in this field, because the moving body needs solidity to push off from but experiences varying degrees of difficulty in passing through it. Further categories of the topology of Field 1 include: inside/outside the body, along with degrees of withinness and withoutness, and the threshold between them; separation/connection between bodies, between bodies and things, between and among things. [3]

In this passage, Burrows explains that humans understand themselves as centers traversing space. Not only that, our sense of reality relies on this notion of being a center. The visual sense dominates by assisting the body to move and navigate through "solidity." However, sound and hearing play a role here. Sounds surround us, while objects of vision only lay ahead of us; they drive us forward. We can hear sounds behind us, but we cannot see them. Thus it is sound that brings sophistication to our notion of centripetal/centrifugal action, "withinness and withoutness" driving the body's trajectory.

AR as a medium is only on the cusp of realizing the human's conceptual centric placement in the interface. There are certainly some inventions that strive to meet this design goal, but we need more work in this area.

In 2005, Marleigh Norton and Blair MacIntyre presented at ISMAR a very simple game called Butterfly Effect which they identify as " a 3D puzzle game using augmented reality" [21]. One unique aspect of this interface is user positioning:

The goal of this project is to explore the design of an interesting AR game that leverages the structure of the physical world during gameplay without requiring the computer to have a detailed model of the world. . We focus on this issue because AR games are not likely to be feasible any time soon if they require accurate, detailed models of the world. In Butterfly Effect, the player is presented with a widely distributed collection of virtual butterflies in a 3D volume around her. The game is played in any physical space that the player wishes; her real home, office, backyard, or park. The player moves around freely in her environment, collecting the butterflies by moving near them. [21]

These designers position the human participant in the center of the interface and arrange the virtual component to surround her. They argue that by leveraging the structure of the physical world, they do not have to provide a "detailed model" of the world for gameplay. In terms of a design strategy, this positioning is also valuable because it centers the player in her hybrid real and virtual world. She is center, the virtual butterflies are periphery, and wherever she wanders becomes the context that she defines with her centricity.

The design principle behind Butterfly Effect widens the scope of possibilities for virtual interaction in other ways. The designers write:

The key challenge arises because the butterflies are scattered in all directions. For butterflies that are out of reach, the player can rotate the virtual world in 90 degree chunks about an axis she defines with her "Tornado Stick" [21]

Butterfly Effect embeds itself in an unknown landscape rather than a known one. With many common conventional first-person shooter games, the designer creates a tunneled landscape for players, which directs their activity within the limited scope of a visual gaze. With Butterfly Effect, no such tunnel exists. The intrigued user defines a strategy for dealing with the scattered butterflies on her own.

Butterfly Effect is an interesting game that clearly pushes technological boundaries, but it is also an exemplary prototype for AR usability because of its minimalist design ideas that complement human physical activity. While it is geared to gameplay, there is no reason why it could not serve as a concept for a platform underlying other domains.

\subsection{Thought Activity}

Humans think. Humans participate with their own thoughts but also with the thoughts of others through systems like speech. While Field 1 is the here and now, "the other, older range of human awareness, we touch, smell, taste and see things all in a vivid solitude"[3], Field 2 is the "meta-sensory range of awareness:”

Field 2 is no passive container for human thought. It has the character of a chronically unanswerable metaquestion drawing out of remarks, conjectures, questions proper (at moments when the metaquestion comes into sharp local focus), exclamations, the unspoken versions of all of these in a compulsive, unceasing stream that is still there, flowing underground, when we dream. The existence of Field 2 encourages a pervasive background conviction that there has got to be more to it all than the This the senses report - because each time we speak or simply imagine there is more, a reality beyond appearance. [3]

Field 2 activity can be explained in simple terms. Burrows describes how thinking can feel detached from the matter-based world. One can imagine the Arctic alongside the Antarctic in one fleeting thought even though they are literally polar opposites. One never senses them in unison, but we can imagine them.

Burrows is really getting at the idea of dynamic interactivity. He explains ways that we interact with other people through speech, but also the ways we negotiate with our own thoughts, dreams, and imaginations. Vision (things that we see) limit the possibilities for interaction, while sound (things that we hear) has an emancipatory role for the imagination. It leads us to "imagine there is more, a reality beyond appearance" [3]. We can transform Burrows' notion of interactivity into a design goal. While many AR applications are firmly rooted in the visual through graphical overlay, for example, there is no reason that we cannot use sound for imaginative or thought-provoking purposes in order to achieve better integration between the real and virtual. 
Research has gone into how sound can assist sight in AR. ZhiYing Zhou et al. argues that 3D sound is an important complement to the visual aspect of AR environments for many reasons, including the fact that it makes it seem more realistic, adding to the immersive feeling of "being inside the AR environment” [22]. By reflecting on Burrows' theory, this paper takes the argument another step and suggests that sound is a gateway not only to a sense of immersion, but also to creative and imaginative experiences, which ultimately make us feel more connected to or integrated with our reality.

Many new media content creators are emerging which claim to provide immersive locative media experiences. Mscapers.com in conjunction with HP Labs for example, offers downloadable context-aware guided tours. Yosemite Walk to the Falls has been developed by HP and the Yosemite Association; it augments a park tour with "a multimedia experience filled with nature information, park history, and a few fun facts” [23]. Conceptually, a tour guide takes a person across a landscape, telling and showing him/her extra information at certain geographic locations. Some media creators, however, leverage not only the real experience as the interactive component, they leverage the imagination through sound rather visuals in the form of multimedia.

The Voices of Oakland project has been developed by the Augmented Environment Lab (AEL) at Georgia Tech and much has been written about it [24][25]. It is an exemplary AR user experience that inspires the imagination by using sound to extend the fixedness of sight through a tour of a local cemetery. The Oakland cemetery is a special historic site in Georgia, where many Atlanta settlers rest, buried there as early as 1850 . Using a GPS system, headphones, and other wearable technologies, visitors range across the cemetery and hear the voices of the dead as they approach particular gravesites. As an augmented reality application, it makes full use of the senses. Wandering close to Carrie Berry Crumley's grave, for example, will let one hear a story from the civil war told from the point of view of a character who was there. She says, "The Federals came down from Tennessee in a relentless march that summer. Our soldiers held them up here and there, but couldn't turn them back" [26]. She accounts for aspects of her life in snippets of tales told by her.

Sight is a springboard for sound. Things that one sees - the cemetery, the grave, the gardens - limit imagination to a local visual context. However, things that one hears, free the imagination to create ones own sense of an authentic experience. We imagine what this character looked like based on her voice and we frame for ourselves a view of settler life. Immersed very much in the real world of trees, dusty pathways, and grass, the Voices of Oakland visitor wanders the real landscape listening to the virtual component in a fully integrated way. If these designers had visually depicted a battle from the civil war in a realistic way, they would not leverage the imagination. These events would have been pinned down to the lowest visual common denominator.

The project is interesting, too, because it has been methodically theorized using humanities-based resources and is thus governed by a design strategy woven with human concepts and rhetoric. Some of the inventors explore Voices of Oakland, their AR installation in terms of Walter Benjamin's notion of aura. Aura relates to authenticity or those feelings of reverence that we feel toward art:

[Aura is] the sense of the 'here and now' that each such work possesses because of its history of production and transmission. This uniqueness lends to each painting or sculpture a special quality, which can in turn evoke an attitude of reverence on the part of the viewer. [7]
They argue that Voices of Oakland creates a sense of "being there" [7] because of careful consideration to aura and authenticity. The ambition is not to recreate the experience realistically, but to have participants share in the drama of the cemetery as an art piece that relates to history.

The Voices of Oakland project is a great design exemplar for other AR interfaces. It brings to life a seemingly mundane landscape in order to reveal its rich historical context through spoken augmentation. In keeping with Burrows' notions, it acknowledges that humans see themselves as physical centers and it refuses to privilege the landscape as the controlling element. It casts participants in the chilling role of hearing the dead tell a tale in a one-on-one dramatic experience. It taps the emotional function that sound plays when it comes to history and culture; telling is so much more personal that seeing. Finally, Voices of Oakland project is a fascinating example of humanities-driven design that yields a human-centric interface.

\subsection{Existential Activity}

Burrows' phenomenology of sound and thought involves existential action, or Field 3 activity [3]. Music is one indicator of it:
Music is the centering of awareness. The more centered awareness is, the more everything in awareness becomes a function or an aspect of the center. And music centers awareness in an object with no sharply defined utilitarian location in the world: the more centered awareness is in such an object, the more does that object come to be provisionally identified with the totality of the world ... When this is carried still further, everything becomes center, and this is the defining condition for Field 3. [3]

Field 3 activity occurs everywhere and nowhere. Put another way, with Field 3 activity "everything becomes center," causing everything to be nowhere. When one becomes so focused on an idea, that idea becomes the center that encompasses everything.

For Burrows, music is a powerful catalyst to existential engagement. He writes, "Sound shaped into music is perhaps the most direct way into Field 3, and Field 3 is a way out of 1 and 2 and the strains within and between them. Field 3 forgives mereness and mortality. Action turns back on itself and converges on stasis” [3]. Field 3 is solely centripetal; all mental action flows inward. This field does not isolate a human because there is no fixed point to which someone can become alien. The boundaries of self and other disappear.

Field 3 is difficult to imagine in terms of a computer interface. How can we get an AR application to inspire feelings like those generated by Beethoven's Symphony 9 "Ode to Joy," for example. The key is to realize that Field 3 activity is more about self-centricity than human-centricity. It governs characteristics of the self. Many activities are utterly solitary. We can explain how our favorite food tastes, but each person experiences pecan pie in a solo manner. No one can truly understand another person's sense of pain, be it emotional or physical. The "Ode to Joy" leaves some exalted, and others indifferent or alienated. We can describe our memories to others, but we cannot really share them; they are solitary. When it comes to future applications, the existential aspect holds the most revolutionary potential for the medium. As technologists march forward, we need to consider what the human or self-centric impact will engender.

Many highly ambitious applications sit just over the horizon in terms of their emergence. One example is the much-touted mind control software and EPOC ${ }^{\mathrm{TM}}$ device by Emotiv [27], which enables one to use brainwaves to move virtual objects on a screen with a headset device. What is interesting about this Brain- 
Machine Interface (BMI) [28] as concept is not the intended gaming application (although very interesting to some!), it is the expectation that these interfaces are to be common in the future, a common aspect of everyday life. In an interview, one Emotiv executive says "We see it becoming a totally ubiquitous device, allowing you to interact in a seamless way with everything else in the world." [29] While this is a lofty marketing claim on the part of a company representative, it also demonstrates a deep-seated desire. People want to augment their lives using technology in increasingly seamless and personal ways. The concepts behind this project imply a high-degree of self-centricity because the computer will have to learn how a person thinks, which a great reversal. Generally, we have to learn how a computer thinks (e.g., Windows operating system).

Other future applications signifying a high degree of selfcentricity involve augmented memory. Using AR to help people remember is not a new concept and many inventors have been involved with it for a long time [30] [31]. However, some applications rely on the concept of remembering our entire lives. The SenseCam device and the MyLifeBits project led by Gordon Bell at Microsoft Research implies that one can store life as a volume of digital memories [32][33]. The idea of interacting with ones memories has great potential, as long as the act of remembering is treated in human terms. Some analysis has suggested that MyLifeBits as a concept treats memory in terms of storing files, rather than understanding memory as a human act. [34].

Field 3 activity, the existential aspect, oversees how we understand ourselves as individuals, as selves, in a manner that cannot be shared with another individual. Furthermore, existential engagement is very much a process across the fields, or a culmination of attention within fields 1 and 2. The physical and mental experiences act as catalysts to the existential.

\subsection{Three Fields as a Process of Human Action}

This section offers a simple example of how the three fields can function to assess a user experience in terms of human action.

An Apple iPod (or any MP3 player for that matter) is not generally considered an AR device, but it is a personal computing device. In terms of human physical movement, the iPod is very successful. Liberated from the livingroom sound system, iPod users move hands-free across a chosen path enjoying their music. The large file capacity and tiny hardware make it possible to carry and experience one's entire music collection. One surrounds oneself in music, fulfilling centric positioning. iPods take great pains with Field 1 design.

When it comes to Field 2, dynamic interaction generated through speech, conversation, thought, or text exchanged with other people, the iPod seems to fall short and ignore it. Subway goers don iPods, for example, to block out subway noise or the dizzying bits of conversation meant for other riders. However, this experience tricks the user into believing that the immersive surrounding audioscape generated by the iPod is a personal place. By diminishing the capacity to interact with other people or the sounds of the environment, the iPod serves as a catalyst to Field 3 activity. While the user sees a socially-shared landscape in the subway train (a space that intrudes on his personal space), he hears a place governed by him by listening to the songs of his choice. In terms of sound, "everything becomes center" [3] his center, which is a condition of Field 3. As a personal computing device, the iPod modulates activities across the fields to create an enjoyable personal experience.

Technology development is at times geared to work-centric or task-centric goals, rather than human-centric ones. That is, we need applications to help with very specific work tasks and we design them according to the task. This paper, however, suggests users constantly try to integrate themselves within contexts. They manipulate reality physically, socially, and existentially at all times. Ultimately, we need to design according to a more complex understanding of human action. There is great leeway for further reflection in this area in order for AR to meet its great potential for ambitious applications.

\section{CONCLUSION}

Let us return to Spook Country and Gibson's dystopian portrayal of society [1]. The word, spook comes the German spōk for "ghost." Ghost comes from geist meaning a disembodied soul, an entity that cannot reconcile its presence in a reality, a thing that belongs elsewhere. Indeed, this paper has implied that technology that neglects to root design concepts in the way humans think about their actions might frame us as spooks or alienated subjects.

This short paper has offered a conceptual model for AR user experiences that might enable a higher degree of human-centricity in order to encourage a more integrated experience between real reality and virtual reality, between humans and machines. It uses a humanities-driven approach by analyzing David Burrows' phenomenology of sound and thought [3], boiling down to a discussion of the three fields of human action relating to body, mind, and self. It suggests that AR designers ought to consider how humans simultaneously act across these three fields even when they are inventing a very specific application. Put more simply, this paper suggests that human-centric design must reflect how people envision themselves.

\section{REFERENCES}

[1] W. Gibson. Spook Country London: GP Putnam and Sons, 2007.

[2] D. Itzkoff. Spirits in the Material World. Sunday Book Review The New York Times 26 August 2007.

[3] D. Burrows. Sound, Speech and Music. Amherst, MA. University of Massachusetts Press, 1990.

[4] S. Mann., with H. Niedzviecki. Cyborg: Digital Destiny and Human Possibility in the Age of the Wearable Computer. Toronto: DoubleDay, 2001.

[5] M. Heim. Virtual Realism. New York: Oxford UP, 1998.

[6] J.D. Bolter and R. Grusin. Remediation: Understanding New Media. Cambridge: MIT P, 1999.

[7] J.D. Bolter, B. MacIntyre, M. Gandy, P. Schweitzer. New Media and the Permanent Crisis of Aura. Convergence: The Journal of Research into New Media Technologies Feb 2006, Vol. 12 Issue 1, p21-39

[8] G. Teyssot. Hybrid Architecture: An Environment for the Prosthetic Body Convergence: The Journal of Research into New Media Technologies Winter 2005, Vol. 11 Issue 4, p72-84.

[9] I. Pedersen. A Semiotics of Human Actions for Wearable Augmented Reality Interfaces. Semiotica Vol. 2005 (155 - 1/4) pp. $183-201$.

[10] I. Pedersen. Mobility, Human-centricity, and the Design of Wearable Augmented Reality Interfaces. International Journal of the Humanities 2006. Vol. 3 No. 1 pp. 143-154.

[11] I. Pedersen. Dehumanization, Rhetoric, and the Design of Wearable Augmented Reality Interfaces. In Small Tech: The Culture of Digital Tools. Eds. Byron Hawk, David Rieder, and Ollie Oviedo, University of Minnesota Press, 2008.

[12] B. Shneiderman. Leonardo's Laptop: Human Needs and the New Computing Technologies. MIT Press, 2003.

[13] R. Azuma. A Survey of Augmented Reality. Presence, Special Issue on Augmented Reality 6.4 (1997): p 355-385.

[14] R. Azuma, Y. Baillot, R. Behringer, S. Feiner, S. Julier and B. MacIntyre. Recent Advances in Augmented Reality. IEEE Computer Graphics \& Applications 21.6 (2001): pp. 34-47. 
[15] M. Heim. The Metaphysics of Virtual Reality, Oxford University P, 1993.

[16] R Coyne. Designing information technology in the postmodern age: From method to metaphor. Cambridge MA: MIT Press, 1995.

[17] P. Dourish. Where the Action is: The Foundations of Embodied Interaction.MIT Press, 2004.

[18] R. Arnheim. The Power of the Center. 1984. 2nd ed. Berkeley: U of California P, 1988.

[19] M. Johnson. The Body in the Mind: The Bodily Basis of Meaning, Imagination, and Reason. Chicago: University of Chicago P, 1987.

[20] G. Lakoff and M. Johnson. Metaphors We Live By. Chicago: University of Chicago P, 1980.

[21] M. Norton and B. MacIntyre Butterfly Effect: An Augmented Reality Puzzle Game, Proceedings of the International Symposium on Mixed and Augmented Reality (ISMAR'05). 212- 213, October 2005.

[22] Z. Zhou, A.D. Cheok, Y. Qiu, and X. Yang. The Role of 3-D Sound in Human Reaction and Performance in Augmented Reality Environments. IEEE Transactions on System Man and Cybernetics Part-A Systems and Humans. 37 (2): 262-272 MAR 2007.

[23] Yosemite Walk to the Falls accessed on 19 May 2009 at www.mscapers.com.

[24] S. Dow, J. Lee, C. Oezbek, B. Maclntyre, J.D. Bolter, and M. Gandy. Exploring spatial narratives and mixed reality experiences in Oakland Cemetery. In Proceedings of the 2005 ACM SIGCHI international Conference on Advances in Computer Entertainment Technology 51-60 (Valencia, Spain, June 15 - 17, 2005).

[25] S. Dow. User engagement in physically embodied narrative experiences. In Proceedings of the 6th ACM SIGCHI Conference on Creativity \& Cognition 280-280 (Washington, DC, USA, June 13 15, 2007).

[26] “Carrie Berry Crumley” Voices of Oakland accessed 19 May 2009 at http://www.cc.gatech.edu/projects/ael/projects/oakland/1oakland02.htm.

[27] T. Le. The Brain: revolutionary interface for next-generation digital media. In Proceedings of the 4th international Conference on Foundations of Digital Games (Orlando, Florida, April 26 - 30, 2009).

[28] B. S. Minnery and M.S. Fine, M. S. Neuroscience and the future of human-computer interaction. interactions 16, 2 (Mar. 2009),

[29] D.H. Freedman Reality Bites. Inc. December 2008. accessed Tuesday, May 12, 2009 At http://www.inc.com/magazine/20081201/reality-bites.html.

[30] B. Schiele, T. Starner, B. Rhodes, B. Clarkson, and A. Pentland. Situation Aware Computing with Wearable Computers. Fundamentals of Wearable Computers and Augmented Reality. Eds. Woodrow Barfield and Thomas Caudell. New Jersey: Lawrence Erlbaum Associates, 2001. 511-537.

[31] R. DeVaul, S. Schwartz, and A. Pentland. The Memory Glasses Project. 5 December, 2000. MIT Media Lab. 28 October 2003. accessed 19 May 2009 at http://www.media.mit.edu/wearables/mithril/memory-glasses.html.

[32] G. Bell and J. Gemmell A Digital Life. Scientific American. Mar 2007, Vol. 296 Issue 3, 58-65.

[33] J. Gemmell, G. Bell, and R. Lueder. MyLifeBits: Personal Database for Everything. Communications of the ACM 49.1 2006. 89-95.

[34] I Pedersen. MyLifeBits, augmented memory, and a rhetoric of need. Continuum: Journal of Media and Cultural Studies. June 2008, 22:3, pp. 375 - 384. 